\title{
Editorial
}

\section{Biomass Materials for Metallurgical Applications}

\author{
Liming Lu $\mathbb{D},{ }^{1}$ Xianchun Li, ${ }^{2}$ Merrick Mahoney, ${ }^{3}$ and Zhiqiang Zhang ${ }^{2}$ \\ ${ }^{1}$ CSIRO Mineral Resources, Technology Court, Pullenvale, QLD 4069, Australia \\ ${ }^{2}$ University of Science and Technology Liaoning, Anshan, Liaoning, China \\ ${ }^{3}$ Newcastle Institute for Energy and Resources, University of Newcastle, University Drive, \\ Callaghan, NSW 2308, Australia \\ Correspondence should be addressed to Liming Lu; liming.lu@csiro.au
}

Received 25 February 2018; Accepted 25 February 2018; Published 27 May 2018

Copyright (c) 2018 Liming Lu et al. This is an open access article distributed under the Creative Commons Attribution License, which permits unrestricted use, distribution, and reproduction in any medium, provided the original work is properly cited.

Steels are by far the most widely used of metallic materials and will continue to be vitally important to our society. However, production of steel from virgin raw materials is currently energy intensive, requires substantial use of fossilbased carbon sources, such as coal and coke, and results in high $\mathrm{CO}_{2}$ emissions. Based on the statistics available, worldwide steel production is believed to contribute to about $5-6 \%$ to total world greenhouse gas emissions. Hence there have been considerable efforts over the years in examining the use of biomass-based materials in primary metal production as a fuel and reductant in place of fossil fuel carbon sources. The biomass material is not only $\mathrm{CO}_{2}$ neutral but also likely contains less $\mathrm{S}, \mathrm{N}, \mathrm{Cl}$, and other heavy metal elements, which are harmful to both the environment and human health as well as metal quality.

The special issue on the biomass materials for metallurgical applications is dedicated to the recent advances in the characterisation, processing, and utilisation of biomass materials for metallurgical operations. Among the six papers collected, four papers endeavoured to develop new iron orecarbon composite pellets in which biomass materials are used as a reductant, while the other papers studied the possibility to use biomass materials as an alternative fuel in the sintering processes. The papers evaluated a wide range of biomass materials from raw biomass such as cow manure and wood to pretreated biomass materials such as biochars from rice husk, peanut shell, oil palm empty fruit bunch (EFB), and acutissima.

Clearly, we still have a long way to go for large-scale applications of biomass materials in metallurgical industries. First, the biomass materials need to achieve the qualities ideally suited to their potential applications, such as iron ore sintering and blast furnace iron making. In addition to the quality suitability of biomass materials for metallurgical applications, the availability and cost of biomass materials must also be considered. The availability and cost of biomass materials are two key factors hindering the introduction of biomass materials in metallurgical industries. More studies concentrating on the availability and possible production chain of biomass materials for metallurgical applications are urgently needed in order to develop and manage the biomass sources on a sustainable basis and to develop a high-capacity production technology at a low cost and less environmental impact.

Liming $\mathrm{Lu}$

Xianchun $\mathrm{Li}$

Merrick Mahoney

Zhiqiang Zhang 


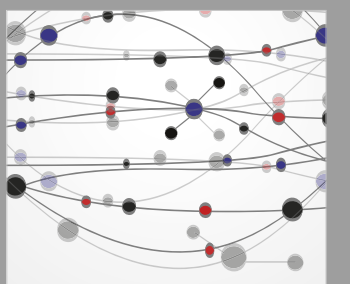

The Scientific World Journal
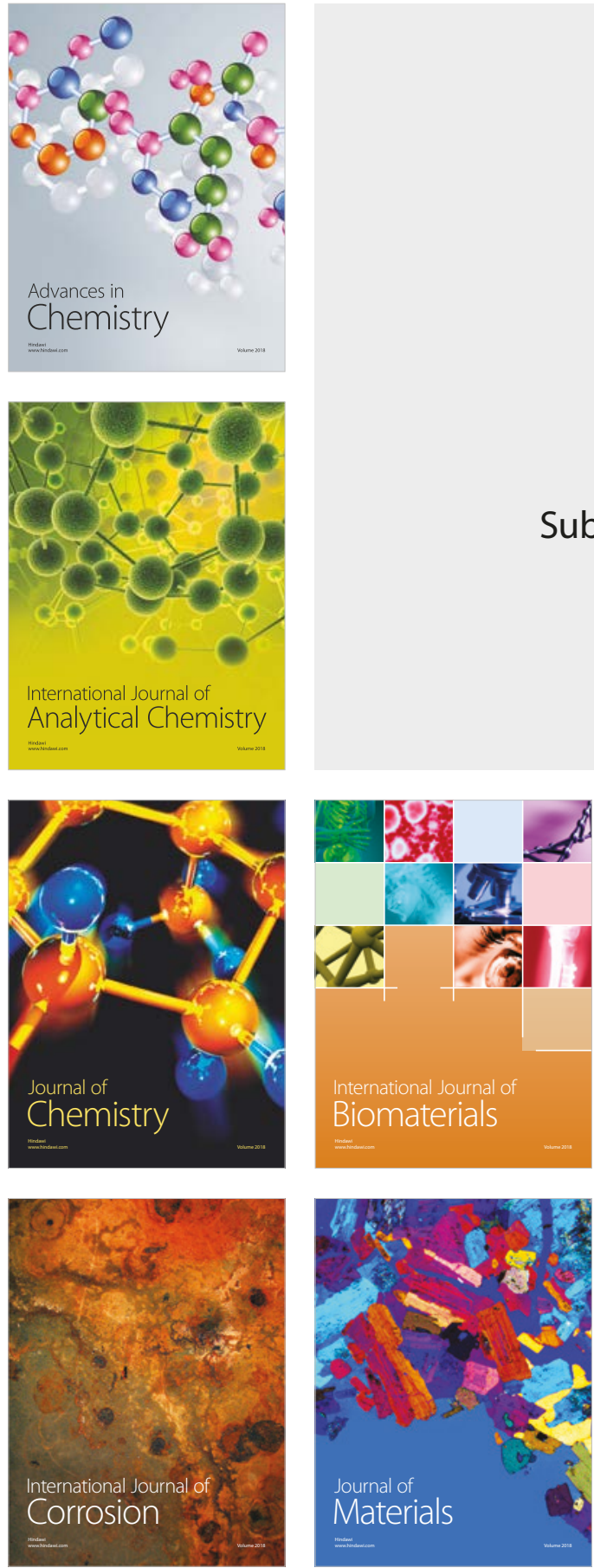

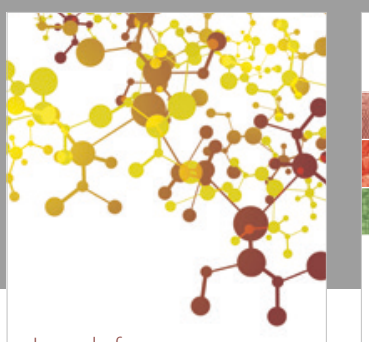

Journal of

Applied Chemistry
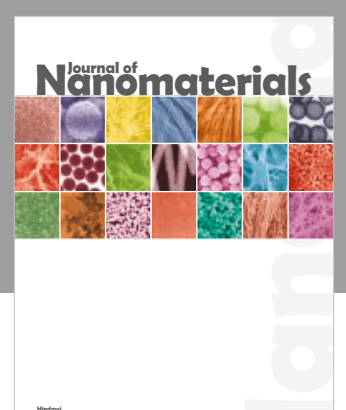

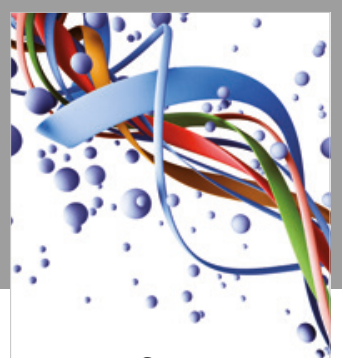

Scientifica

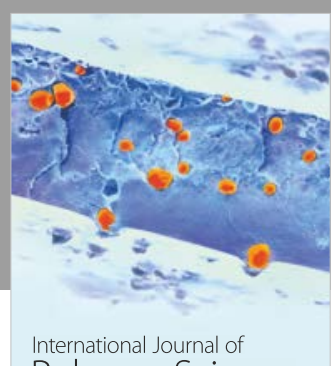

Polymer Science

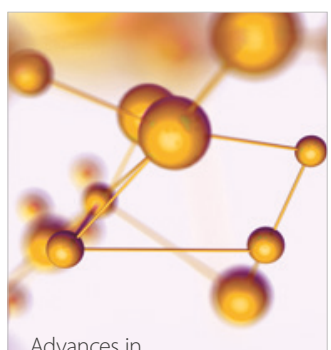

Physical Chemistry
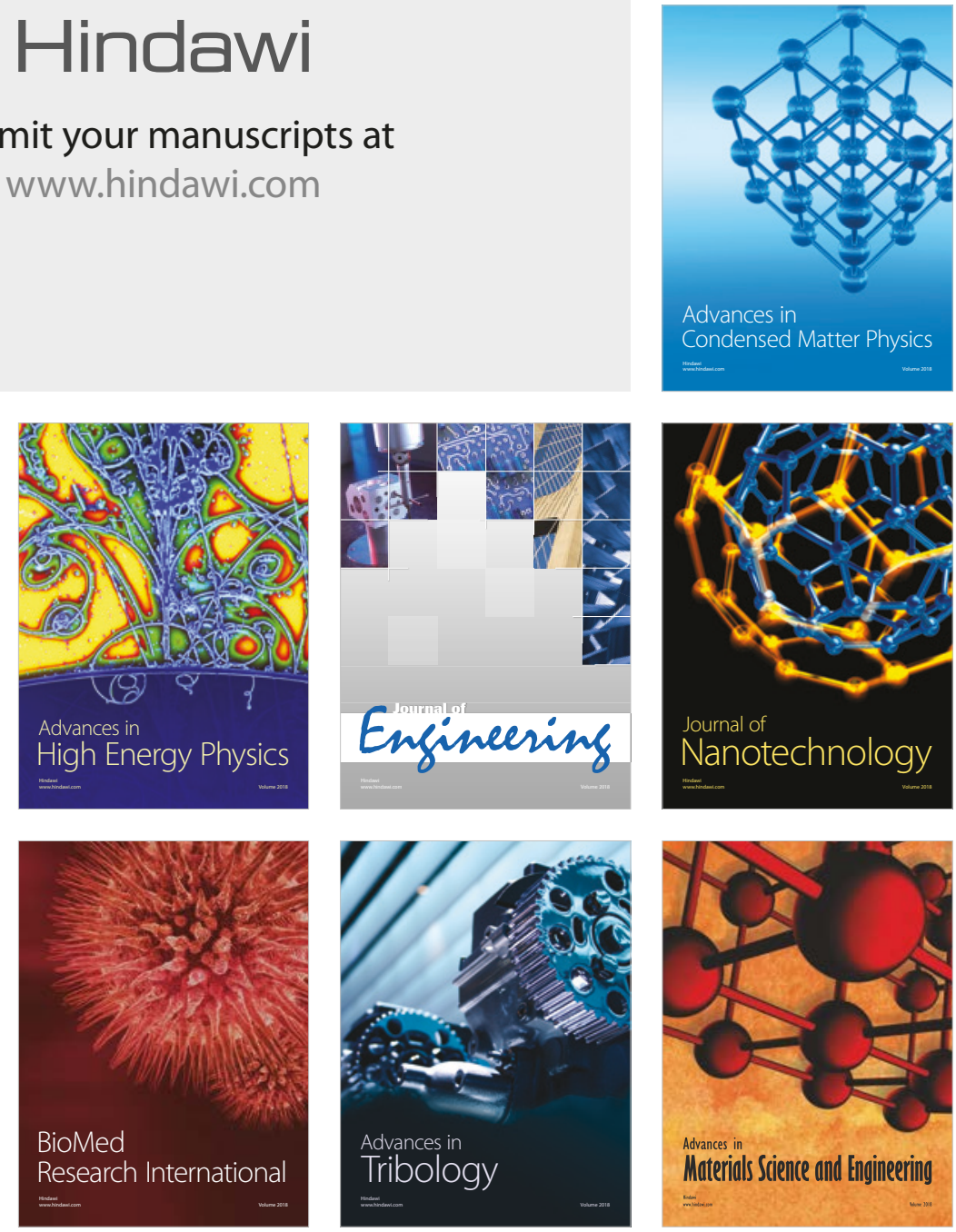